\title{
Design and Synthesis of New Phosphoramidates Chloroquine Analogs
}

\author{
Leandro F. Pedrosaa ${ }^{a}$ Antônia C. R. Furtado ${ }^{a}$, Carolina T. Machadoa ${ }^{a}$ Raphaela de M. \\ Baêsso ${ }^{a}$, Neidemar de M. S. Bastos, William P. de Macedo ${ }^{a b}$, Marcos C. Souza ${ }^{a *}$ \\ a Universidade Federal Fluminense, Departamento de Química Orgânica, 24020-150, Niterói, RJ. \\ ${ }^{b}$ Universidade Estadual de Goiás, UnUCET, 25000-000, Anapolis, GO. \\ ${ }^{*} e$-mail corresponding author: gqomarc@vm.uff.br
}

Keywords: Antimalarial, chloroquine, phosphoramidate.

\section{INTRODUCTION}

About 3.3 billion people - half of the world's population - are at risk of malaria. Every year, this leads to about 250 million malaria cases and nearly one million deaths. ${ }^{1}$

Therefore, there is urgency to develop new affordable, safe, and efficacious antimalarilas. Although the resistance to chloroquine (CQ) and related 4-aminoquinoline antimalarial drugs has emerged; designing new antimalarial based on the quinoline pharmacophore has distinct advantages due to unique pharmacological effect of 4aminoquinoline drugs. ${ }^{2}$

Several studies demonstrated that various structurally diverse modifications in the side chain of $\mathrm{CQ}$ were well tolerated for the antimalarial activity. Systematic variation of the branching and basicity of the side chain of $C Q$ yielded the new 4aminoquinoline derivatives exhibiting excellent potency against CQ-sensitive and CQ-resistant strains. ${ }^{3}$

A new class of Chloroquine analogs containing phosphoramidate group and different alkyl spacer (Figure 1) was structurally planned by modification of side-chain based on the previously described.

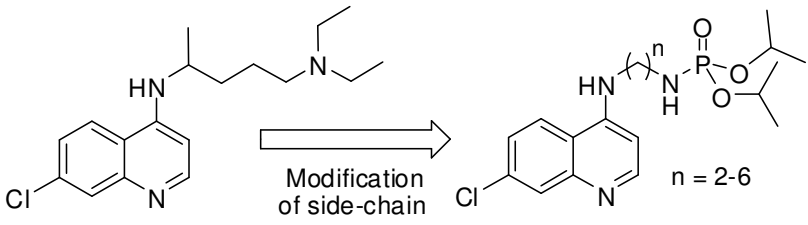

Chloroquine of side-chain

Figure 1. Design strategy for Chloroquine analogs (1).

\section{RESULTS AND DISCUSSION}

The phosphoramidates chloroquine analogs (1a-e) used in the present study were prepared by nucleophilic aromatic substitution on 4,7dichloroquinoline (5) with excess of aminoalkyl phosphoramidates (4a-e) in solvent-free at $110^{\circ} \mathrm{C}$ overnight as outlined in Scheme 1.
The starting aminoalkyl phosphoramidates (4a-e) are easily prepared from selective monophosphorylation of aliphatic diamines (3a-e) with diisopropyl phosphonate (2). ${ }^{4}$

All the synthesized compounds were well characterized by $\mathrm{IR}, 1 \mathrm{H}, 13 \mathrm{C}$ and $31 \mathrm{P}$ NMR spectroscopy.
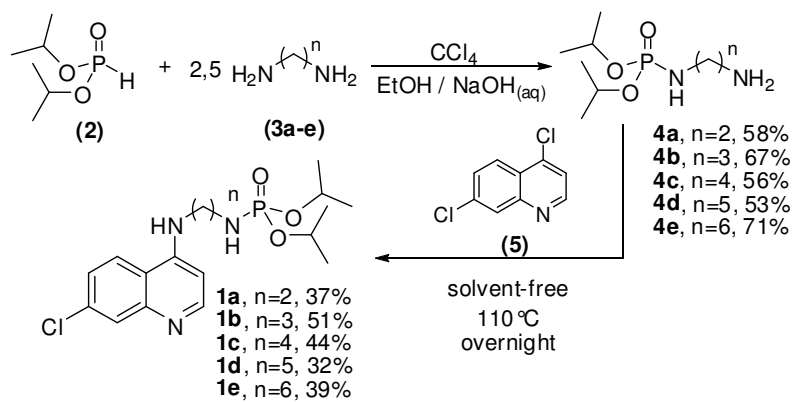

Scheme 1. Synthetic route of new phosphoramidates chloroquine analogs $(\mathbf{1} \mathbf{a}, \mathbf{b})$.

\section{CONCLUSION}

Five new phosphoramidates derivatives, closely mimicking the antimalarial drug chloroquine, have been prepared.

The compounds were obtained in satisfactory yields by nucleophilic aromatic substitution of the chloro atom in 4-position of the 4,7dichloroquinoline (5) with aminoalkyl phosphoramidates (4a-e).

Studies on the properties antimalarial of these new derivatives are currently under investigation in our laboratories.

\section{ACKNOWLEDGEMENTS}

UFF, FAPERJ, CAPES and CNPq.

\section{REFERENCES}

${ }^{1}$ WHO, World malaria report: 2010.

${ }^{2}$ Kumar, A.; et al., Bioorg. Med. Chem. Lett., 2010, 20, 7059

${ }^{3}$ Yearick, K.; et al., J. Med. Chem., 2008, 51 (7), 1995.

${ }^{4}$ Souza, M. C.; et al., Phosphorus, Sulfur, and Silicon, 2006, 181, 1885 